\title{
The apparent paradox of language evolution: can Universal Grammar be explained by adaptive selection?
}

\author{
Manfred Bierwisch
}

\section{Three introductory remarks}

The evolution of natural language has again become the subject of lively debate. Like in previous discussions of this topic, an important distinction is often ignored: the origin of both a given language and of languages in general is not the same as the origin of the language capacity as a speciesspecific disposition. The corresponding developments must be clearly distinguished.

\subsection{Language and language capacity}

Ferdinand de Saussure (1916) draws a crucial distinction between langue, parole, and langage. For Saussure, the faculte de langage is the speciesspecific capacity to acquire and use a natural language; it is a biological property of the human organism. Langue refers to natural languages like French, Hungarian, or Hebrew; it is a social institution that consists of the knowledge shared by the members of a given speech community. Parole refers to the intentional use of linguistic expressions and comprises the actual, psycho-physical processes determined by the knowledge of a language and by other situational conditions.

Noam Chomsky (1965) employs the terms language capacity, linguistic knowledge or competence, and language use or performance to draw similar distinctions, though in a slightly different way. Whereas Saussure is primarily concerned with the socio-cultural aspects of language as an institution (in other words: with langue), Chomsky is essentially interested in the mental structure of individual speakers' knowledge. For Chomsky, language as a social institution depends on there being sufficient similarity between speakers' knowledge. For even if language as a social institution is not merely the linguistic knowledge shared by the members of a given speech community, individuals' knowledge remains an indispensable condition. 


\section{Manfred Bierwisch}

We will see that the asymmetrical interdependence between Saussure's emphasis on language's social aspect and Chomsky's interest in language's mental structure will have intriguing consequences for the issues related to phylogenetic development. Both Saussure and Chomsky view the language faculty as an inherited biological condition and the use of language as a process carried out by individual, psycho-physical mechanisms operating under various conditions.

To suggest how linguistics can contribute to the understanding of language phylogenesis, we need to make the above concepts slightly more specific. Following Chomsky (1986), this can be done along the following lines. The formal structure of the human language capacity that organises possible systems of linguistic knowledge is called Universal Grammar (UG). The system of knowledge that determines a possible natural language (L), the "internal language" L, is characterised by the Grammar $(\mathrm{G})$ of $\mathrm{L}$. The structure of a given utterance $(\mathrm{u})$ determined by $\mathrm{L}$ is characterised by the structural description (SD) of u. UG, G, and SD are theoretical constructs corresponding to or describing mental structures that are schematically related in the following way:

$$
\begin{aligned}
& \Longrightarrow \text { UG } \begin{array}{l}
\Longrightarrow \\
\Longrightarrow \text { ontogenesis }
\end{array} \quad \text { G } \begin{array}{c}
\Longrightarrow \\
\text { actual genesis }
\end{array} \\
& \text { (language origin) (language acquisition) (language use) }
\end{aligned}
$$

The processes indicated by the arrows are, of course, fundamentally different. First, language use - primarily the production and comprehension of utterances - consists of short-term processes lasting seconds or less. They are relevant because they are the source of all primary linguistic data. One might furthermore argue, like Hubert Haider (1991), that UG's properties are essentially due to the processing routines by which humans deal with linguistic utterances.

Second, the language acquisition process is part of individual human organisms' (presumably epigenetic) development. It is relevant because it depends (in addition to the actual experience a child is exposed to) on the language capacity - that is, UG. UG renders the process possible and thus shapes the structure of the resulting knowledge.

Third, the phylogenetic process from which UG originates is subject to biological evolution. Yet the origin of UG should obviously not be confused with the origin of a primordial language. The former is part of biological evolution, whereas the latter is part of cognitive or social evolution. Nearly 
all speculations about language origin - including those of Plato, Leibniz, Herder, and Engels - either address the origin of language knowledge (usually: words) or simply confound the two issues.

\subsection{Evolution}

Language capacity - and thus UG as its structural aspect - is a species-specific trait of homo sapiens. In other words, its genetic foundation must reside in the 1.5 percent that, according to a recent assessment, distinguishes the human genome from that of our closest relatives. Like any other genetically determined trait, language capacity is the result of evolution. Following Gerald Edelman (1987), evolution can be characterised by three conditions. Variation creates randomly alternative properties, the origin of which is not causally connected to the conditions governing subsequent processes of selection. Selection favours certain variants over others during encounters with an independently changing environment. Heredity distributes the favoured variants within a population via differential reproduction or amplification. Variation applies to the individual's genotype, selection applies to its phenotype, and heredity to the population the individual belongs to. The standard Darwinian view conceives of selection as adaptive in the sense that the variants survive via reproduction if they guarantee their bearer a benefit over possible competitors in survival and reproduction, thus spreading the selected property within the population. Selection thus guarantees adaptation according to environmental conditions. This notion of adaptive selection, typically referred to as "survival of the fittest", requires two significant amendments.

First, random variants that do not provide a benefit might survive and be reproduced - without adaptive consequences - as long as they do not represent a manifest disadvantage. Stephen J. Gould (1982) has used the term exaptation to describe this extension of Darwinism. Exaptive properties can, however, become advantageous (or disadvantageous) if the environment subsequently undergoes relevant changes. In other words, between the origin of a variant and what might be called "delayed selection" other properties or conditions may change, thus assigning the variant a different role.

Second, genotype variation might generate adaptive properties that are causally connected to concomitant, but nonadaptive properties. Known as "emergence", this phenomenon might be insignificant, like for example the colour of the iris as opposed to its shape. Of greater importance are, for 
example, certain concomitants of increased brain size. Phenomena of emergence - combined with those of exaptation - are of particular relevance considering the genome's enormous complexity and the largely unknown means by which it determines the equally complex proteome, that is, the system of proteins that are responsible for the phenotype's morphology and behaviour.

With respect to language, there is no reason to suppose researchers will one day identify a discrete group of genes that separately and completely determine nothing but a well-defined, adaptively selected brain structure supporting just the capacity described by UG.

\subsection{Signals and thoughts}

Nevertheless, language capacity and the knowledge it gives rise to comprise a biologically determined mental system that organises a specific component of human behaviour. It interacts with other systems of the mind/brain like vision and hearing as well as locomotion and other motor activities. Even though there are obvious and relevant conditions shared by several of these systems - including linear organisation, hierarchical structure, and invariant patterns retrievable from memory - language capacity has its own, domainspecific properties. Roughly speaking, language capacity recruits two phylogenetically prior systems of mental organisation, thereby creating a systematic correspondence between their representations. The first is the system of articulation and perception (A-P) that underlies the production and recognition of invariant structures of external signals. The second is the system of conceptualisation and intention (C-I) that allows for conceptually organised and intentionally controlled representations of experience.

Both A-P and C-I, which support what is usually termed the form and meaning of linguistic expressions, might in themselves be complex aggregates of mental organisation. A-P is normally instantiated by the systems that control vocal articulation and auditory perception. But recent research initiated by Edward Klima and Ursula Bellugi (1979) clearly demonstrates that sign language, based on the production and perception of visual signals, represents a fully adequate alternative. C-I must be construed as integrating the full range of systems involved in conceptual organisation, intentional control, and motivational instigation of experience and behaviour. Both systems are products of previous evolutionary stages. They might also exhibit new properties, sinee the language capacity exploits their possibilities. 
Schematically, these considerations can be abbreviated as follows:

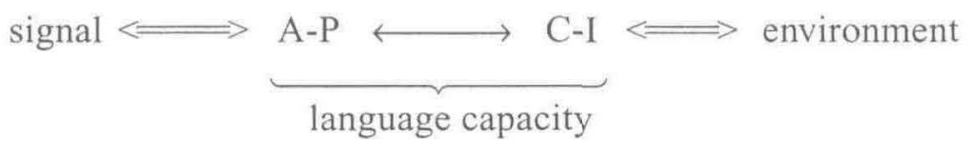

We can also refine things somewhat. The knowledge of $\mathrm{L}$, characterised by $\mathrm{G}$, determines the correspondence between A-P and C-I by means of the phonetic form interface (PF) and the semantic form interface (SF), where $\mathrm{PF}$ and SF represent those aspects of A-P and C-I on which the correspondence between the two domains relies. These refinements yield following schema:

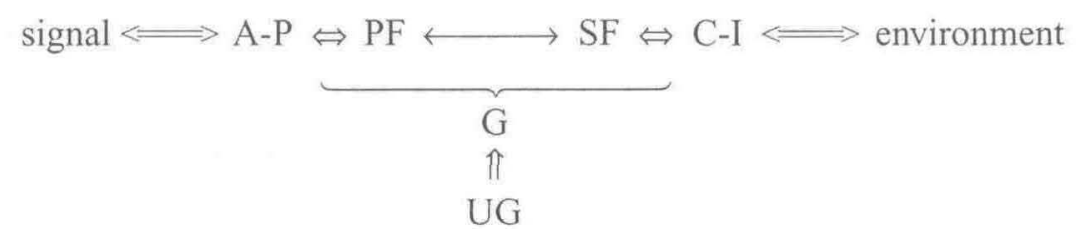

Based on the possibilities provided by $\mathrm{UG}, \mathrm{G}$ determines the relation given by pairs $\langle\pi, \sigma\rangle$, where $\pi$ and $\sigma$ belong to $\mathrm{PF}$ and SF, respectively. The crucial point is that this relation ranges over an unlimited set of pairs. $G$ must therefore be a system for computing new pairs on demand. This has far-reaching consequences.

\section{Properties of UG}

It is an empirical fact that normal human beings acquire the language spoken in their environment on the basis of varying and incomplete input. Yet external input only partially determines the structure and result of this epigenetic process. It must also rely on internal, biologically fixed conditions. UG specifies the structural conditions this internal disposition contributes to the structure and result of the language acquisition process. These internal conditions cannot be inspected directly. They therefore must be identified hypothetically by examining the structure of the observable result. Section 2.1 sketches UG's main traits. 


\subsection{Lexical items and their combination}

As a necessary condition, a system of linguistic knowledge must organise a fairly large set of basic expressions or lexical items (roughly: words), each associating a form $\pi$ (a representation in PF) with its meaning $\sigma$ (a representation in SF). However, for pairs $\langle\pi, \sigma\rangle$ to be able to function as proper linguistic expressions, they must be categorised by grammatical features determining their possible combination into larger expressions. (1) below provides the most elementary illustration of this point. It combines the words daddy and jump in different ways, leading to different categories of expressions:
(1) (a) daddy jumps
(b) daddy's jump
(c) jumping daddy

In (1a), jump is a verb, the final $-s$ indicates the personal inflection, and the whole expression a clause. In (1b), jump is a noun, the $-s$ attached to daddy indicates the possessive case, and the whole expression is a nominal phrase with jump as its nucleus. In (1c), the inflection -ing turns jump into an adjective modifying $d a d d y$, which in this case is the nucleus. The knowledge of lexical items thus comprises three types of information $\langle\pi, \gamma, \sigma\rangle$, where $\pi$ and $\sigma$ represent form and meaning, as already noted, and $\gamma$ constitutes what might be called the "grammatical form" (GF). GF categorises the expression in question and determines its combinatorial properties. Hence, knowledge of English includes lexical items like (2), where /jump/ indicates a feature matrix of PF, JUMP an abstract characterisation of a particular type of motion, and [Verb] and [Noun] the relevant features of GF.

(2) (a) $\langle/$ jump/, [Verb], JUMP $\rangle$

(b) $\langle$ jump/, [Noun], JUMP $\rangle$

(2) (a) and (b) must presumably be treated as one lexical entry, allowing for [Verb] and [Noun] as alternative specifications of GF. These simplified examples indicate that UG must, among other things, provide the following conditions:

(3) (a) Accessibility of primitive elements in terms of which the interface representations PF and SF and the categorisation GF can be specified and fixed in memory.

(b) The general format of lexical data structures comprising $\langle\mathrm{PF}, \mathrm{GF}, \mathrm{SF}\rangle$. 
Three things need to be emphasised. First, the general availability of specific types of primitive elements, their properties, and their organisational format in lexical items are neither obvious nor trivial. They are crucial determinants of the way in which linguistic expressions can be organised. Moreover, they specify the structure of representations supporting the mental computation of complex expressions. Second, GFs like Noun, Verb, Genitive, and so forth determine which lexical information goes beyond the mere association of form and meaning. It is the grammatical information $\mathrm{g}$ that determines the combinatorial possibilities of words and distinguishes them from labelled concepts. Finally, lexical items can be subject to inflection and derivation - the two branches of Morphology -, which is another of GF's facets. Morphological distinctions allow recurrent conditions in the SF-PF correspondence to be explicitly related to and fixed by features of GF. This provides guidelines for the combinatorial aspect of linguistic expressions, as even simple cases like (1) above indicate.

The following constructions with the particle again demonstrate how intricate lexical information and its combinatorial aspects are. Their PF differs only by nuclear-stress placement (marked by capital letters). But the corresponding SF requires different conditions (informally added in parentheses):

(4) (a) Mary had LEFT again. (presupposition: Mary was absent before) (b) Mary had left AGAIN. (presupposition: Mary had left once before)

In other words, again adds the notion of repetition to both assertions about Mary having left. If, as in (4b), again is stressed, it repeats the event of Mary's leaving, whereas if, as in (4a), left is stressed, only the state of Mary's absence is repeated. In (5), the required lexical information is indicated in a rather provisional way:

(5) (a) 〈/again /, [Particle], REPETITION OF P

(b) Content of $\mathrm{P}$ is determined by position and stress.

"REPETITION OF P" indicates that P presupposes that $\mathrm{P}$ was the case before (Stechow 1996 offers a detailed discussion of the complexities of again). I want to emphasise two things. First, knowledge of particles like again, also, or almost involves intricate conditions relating lexical information to the combinatorial requirements of syntactic, semantic, and even phonetic structure. Second, complexities of this sort are by no means 


\section{Manfred Bierwisch}

exotic or marginal phenomena, but belong to the core of linguistic knowledge. Even though their specific details differ from language to language and are subject to language acquisition, UG provides the general possibility of their structure.

\subsection{Hierarchy and compositionality}

The principles by which the combinatorial conditions of lexical items are realised are largely predetermined and subject to highly restricted variation in language acquisition. With respect to $\mathrm{PF}$, combination essentially consists of sequential ordering. For example, John is followed by walks, which is followed by slowly in John walks slowly. Things are less trivial if prosodic consequences - stress and intonation - are considered, but these aspects are still related to the signal's linear organisation. The crucial step is due to SF which imposes an inherently nonlinear, hierarchical structure on the sequential combination of basic expressions. This hierarchy and its constituents create the GF, usually represented by tree structures or bracketings, as illustrated in (6). Here, constituents are categorised by features originating in the GF information of lexical items:

(6) (a)

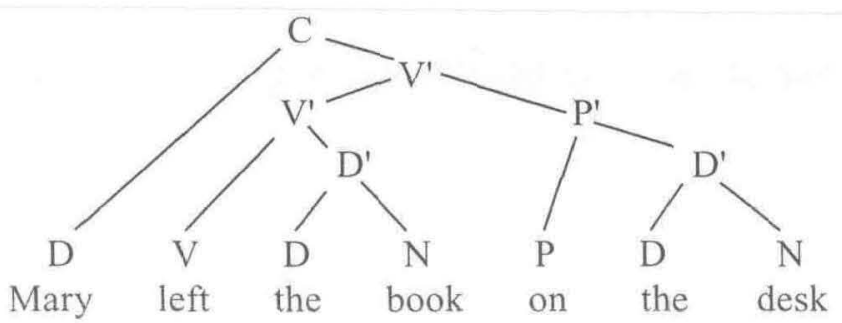

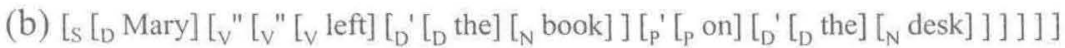

The category symbols (D, N, P, V, C for Determiner, Noun, Preposition, Verb, and Clause and $\mathrm{X}^{\prime}$ for complex categories with the head or nucleus X) are standard abbreviations. Their particular properties need not concern us here. (6a) is equivalent to the labelled bracketing (6b). The principle underlying this type of structure can be characterised as follows: 
(7) Two constituents $X$ and $Y$ combine into a complex constituent $\left[{ }_{\gamma} \mathrm{X} Y\right]$, where the category $\gamma$ is determined by the category of the head.

This formulation is somewhat simplified. In particular, it ignores the nontrivial selectional conditions lexical items impose on potential co-constituents, as illustrated above with respect to again (see Bierwisch 1997 for a detailed discussion).

Example (6) illustrates what is traditionally called Constituency or Phrase Structure. It is an essential factor in the computation by which complex expressions derive their meaning. It gradually integrates the lexical items' SF into the SF of more complex constituents. The SF of the Determiner the therefore combines with the conceptual conditions of the Noun desk, selecting from the (situational) context a particular object that meets just these conditions. The next step turns the individual thus specified into the anchor point of the relation expressed by the Preposition on, which fixes the location of the act ascribed to Mary. More generally, the hierarchy described by Phrase Structure controls the Compositionality of the mental computation, by which a complex expression's SF derives from the SF of its constituents on the basis of their combination. This effect can be observed directly in cases of Phrase Structure differences. For instance, if we replace the verb leave by the verb buy, the natural structure would be (8). Here, on the desk combines with book, adding further conditions to the object in question rather than to Mary's act of buying:

(8) $\left[{ }_{C}\right.$ Mary $\left[{ }_{V}\right.$ bought $\left[_{D}\right.$ the $\left[_{N}\right.$ book $\left[_{P}\right.$ on the desk $\left.\left.\left.\left.]\right]\right]\right]\right]$

In many cases, two alternative structures that determine clearly different meanings. Thus in (9), in which only the relevant differences in constituency are indicated, we get (9a), in which Robert Redford is most likely a protagonist of the film and (9b), in which he is a participant of the discussion.

(9) (a) they [discussed [the film with Robert Redford] ]

(b) they [ [discussed the film] [with Robert Redford] ]

In other words, the structural ambiguity of linguistic expressions can come from an alternative Phrase Structure being assigned to the same PF. 


\section{Manfred Bierwisch}

\subsection{Chains of positions}

A further characteristic property of natural languages seems to go beyond the minimal requirements for the mapping between PF and SF. A constituent's sequential position in PF may not correspond to the role it plays in SF. A characteristic case in point is the position of the finite verb in German, Dutch, and a number of other languages:

(10) (a) daß das Konzert erst eine halbe Stunde später anfing.

(b) das Konzert fing erst eine halbe Stunde später an. ((that) the concert didn't began until a half hour later)

Because anfangen must be registered as a lexical item, the SF of which cannot be derived from that of an and fangen, the two parts of the Verb separated in (10b), fing and an, must be one constituent with respect to SF, as is overtly the case in the subordinate clause construction (10a). Hence, fing has two functions in (10b): an overt position in PF and a covert participation in the Verb's SF. The relevant structure resembles (11), in which fing indicates the covert position; the arrow connects it to its overt realisation:

(11) $\left[_{C}[D\right.$ das Konzert $]\left[_{V}\left[\left[_{V}\right.\right.\right.$ fing $]\left[\left[_{A}\right.\right.$ erst eine halbe Stunde später $]\left[_{V}\right.$ an $\left[{ }_{V}\right.$ f $\left.\left.\left.]\right]\right]\right]$

The principle that leads to chains of positions can be formulated as follows:

(12) A constituent $\mathrm{X}$ can occupy more than one position in a structure $\mathrm{K}$ depending on the grammatical features of $\mathrm{X}$ and $\mathrm{K}$, forming a chain $\left(\mathrm{X}_{\mathrm{n}} \ldots \mathrm{X}_{1}\right)$ of positions, in which only $\mathrm{X}_{\mathrm{n}}$ is realised in $\mathrm{PF}$.

The features on which chain formation depends originate in the lexical items involved. The details are anything but trivial, but need not concern us here.

As cases like (13) show, a complex expression may contain more than one chain, whereas (14) illustrates the fact that chains may be involved in the ambiguity of linguistic expressions: 
(13) (a) Was fängt Peter damit an? (What is Peter doing with that?) (b) [was [fängt [Peter [damit [ [an

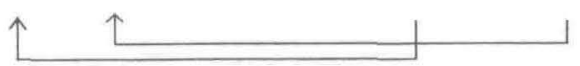

(14) (a) Mary had [plans [to leave] ]

(b) Mary had [plans [to leave ]

In (14a), Mary intends to leave, whereas in (14b) Mary had plans to drop off. Typically, (14a) has nuclear stress on leave, (14b) on plans.

\subsection{The innateness of $U G$}

To sum up, UG must essentially provide the following conditions or principles:

(15) (a) Primitive elements from which the interface representations PF and $\mathrm{SF}$ as well as the categorisation required in GF can be invented; see (3a).

(b) The organisation of lexical items, including morphological indicators; see (3b).

(c) Phrase Structure, which supports SF's Compositionality; see (7).

(d) Chain formation, which assigns one constituent to different positions; see (12).

The above description of UG's content is, of course, simplified. For my purposes here it is important to note that the conditions (15)(a) to (c) - with the possible exception of Morphology - are conceptually necessary for any system generating a correspondence between A-P and C-I that goes beyond a list of pairs $\langle\pi, \sigma\rangle$. By contrast, (15d) is an empirical fact about the human language capacity that does not seem to be logically necessary. In any event, only systems with the above four abbreviated structural principles can support the knowledge and use of a natural language. By the same token, these principles delimit the range of possible natural languages. This includes their geographical, historical, or social variation. 


\section{Manfred Bierwisch}

What is the status of the principles constituting UG? We don't know how genetically determined aspects of complex behaviour are realised in the brain, let alone how the brain's relevant properties are determined by DNA structure. Nevertheless, overwhelming ethological evidence suggests that certain aspects of human behaviour have an inherited and genetically fixed basis. There is no reason to doubt that the language capacity is among them. There seem to be two logical possibilities regarding the more specific assumptions summarised in (15):

(16) Nativist position: UG characterises domain-specific, genetically fixed principles that merely support the ability to acquire and use a natural language.

(17) Empiricist position: UG consists of principles that emerge from the interaction of general mechanisms of association and combination with actual linguistic input.

There are two versions of the nativist position. The maturational hypothesis (see Borer and Wexler 1987) assumes that components of UG become available according to the schedule of (early) ontogenetic development. Pinker's (1994) homogeneity hypothesis assumes that UG is in place from the very beginning. Although recent research into brain maturation seems to render the maturational hypothesis more plausible, both versions presuppose that $\mathrm{UG}$ is genetically fixed.

The empiricist position (see Elman et al. 1996), on the other hand, assumes that the properties of linguistic knowledge emerge from general principles of cognitive organisation. This eliminates the need to stipulate innate conditions specifically supporting the language capacity. The problem with this position is that it either has to claim (contrary to fact) that nonhumans will acquire and use language if exposed to appropriate input or has to include the relevant disposition as a specific component of its general cognitive equipment and its genetic foundation. But this is simply another way of positing what is ultimately a biologically fixed basis for the conditions summarised in (15). 


\section{The evolution of the language capacity}

Assuming that conditions like those in (15) must somehow be fixed in humans' genetic endowment, how could the language capacity have developed phylogenetically?

\subsection{The paradox of adaptive selection}

The theory of adaptive evolution appears to provide a straightforward explanation. A random genetic variation determines a change in brain structure that supports the capacity to produce and comprehend signals that systematically represent complex cognitive structures. Individuals with the ability to communicate verbally have a selective advantage over competitors who lack it. Hence, adaptive selection favours the language capacity, though its genetic basis originated by chance. But this explanation contains a vicious circle.

First, although it does not matter at this point whether (15) correctly characterises the basic traits of this heritage, it is crucial that the genetic basis distinctively accounts for the ability in question. Second, adaptive selection favours this ability if and only if its benefits can be exploited in actual behaviour. This requires at least a limited population to communicate with. The members of this population must therefore already have and use the capacity in question.

In other words, explaining the language capacity by adaptive selection presupposes the property it attempts to explain. The theory of evolution has to cope with this problem for all cases of genetically determined social behaviour. Yet it is of particular intricacy in the case of linguistic communication because the selectional advantage presupposes not only the population whose members previously developed the capacity, but also a language - that is, a system of knowledge based on this capacity - without which the capacity would be of no adaptive value.

The language capacity is an empirical fact, one that is controversial only with respect to its specificity and not to its biological foundation. So there must be ways to avoid the above paradox. In my opinion, there are at least three ways of addressing the issue. They are not mutually exclusive. 


\subsection{Avoiding the paradox}

The first option is to abandon the restriction imposed by adaptive selection and to assume that the language capacity's genetic foundation is due to exaptation (see section 1.2). More specifically, the language capacity and UG's principles sketched in (15) can be genetically fixed and inherited without immediate behavioural consequences. Their full potential will be realised only if appropriate conditions arise. Moreover, the genetic foundation might be a by-product of other (perhaps more general) changes like the relative growth of the brain or the modification of its architecture.

This seems to be Chomsky's (1988) somewhat sceptical position regarding the adaptive explanation. His scepticism is supported by the fact that we know little about the details of genetic information, about the causal structure by which the brain is controlled by the genome, or about the way the brain effects the behaviour. All we do know is that the behaviour exhibits the specific properties illustrated above and that it is species-specific. Furthermore, we know that certain principles of linguistic behaviour (hierarchical structure or the fixing of information chunks in memory) are exploited in domains of behaviour that are not species-specific. Hierarchical structure occurs in various types of motor action, information chunks fixed in memory are crucial for visual perception, and so on. We do not, however, know what aspects of genetically fixed brain structure constitute language's species-specific domain, the domain that computes the systematic correspondence between conceptualisation and articulation.

The second option is to retain adaptive selection and to emphasise the language capacity's cognitive - as opposed to its communicative - benefits. That is, the circularity is removed if the advantage of language does not depend on the behaviour of other members of the population. There is no need to assume that the capacity and its use are already in place, since the advantage only concerns the organism that exhibits the innovation. It consists of the increasing efficiency of cognition (including its far-reaching consequences) brought about by the access to conceptual structures via independently organised and memorised signals. Johann Gottfried Herder's essay on language origin (1772) stresses precisely this cognitive benefit. For Herder, Besonnenheit (reflection) is the crucial property that makes language possible. It not only enables humans to identify invariant characteristics, but also to associate them with reproducible signals. There are two reasons why Herder does not account for the language capacity. First, he is more concerned with naming objects than with addressing the combinatorial aspect. 
Second, he tries to explain the creation of words and takes for granted the capacity to name objects, namely "reflection".

Moreover, the cognitive perspective on adaptive selection shares a significant problem with the communicative approach. In order to establish the relevant advantage, the language capacity must support the spontaneous acquisition and use of linguistic knowledge. Yet this presupposes that a linguistic system is available. True, the cognitive perspective can attribute the creation of such a system to an isolated individual, but this is an artificial assumption that requires justification.

Evolutionary theory seems to offer the third and apparently most plausible option to avoid the paradox of adaptive selection. Evolution, after all, is the cumulative result of tiny steps. The vertebrate eye is the result of numerous minor changes. The same could be true of UG. There are at least two versions of this proposition. Pinker (1994) points out that UG combines a number of more or less self-contained subsystems or modules, like Morphology, Phonology, Phrase Structure, and so forth. These might well be the result of independent developmental steps, of which UG is the sum. Though this model is largely in line with generally accepted principles of evolution, it creates crucial difficulties if adaptive selection is assumed to be decisive with respect to the individual steps. In fact, Pinker runs into the same paradox I discussed above, creating even greater difficulties. Take, for instance, Morphology, the ability to systematically relate grammatical properties to (partial) conceptual interpretation. What is the adaptive advantage of such a capacity if there is, first, no grammatical system for it to improve and, second, no group of speakers whose behaviour is based on the same principles? This paradox applies to any component of UG one might single out for separate evolution. The problem is that though a marginal improvement in an organism's vision yields immediate selective advantages, there is no comparable benefit in acquiring a subcomponent of UG - unless we assume that it generates advantages in other behavioural systems. But then we would no longer be talking about the language capacity.

Bickerton (1995) offers another version of the gradualist position. It differs in two respects. First, the gradual development consists of only a few stages. One of these is a so-called "protolanguage", from which the modern language capacity evolves. Protolanguage (comparable to the first stages of language acquisition or of pidginisation) is a restricted lexical system with a limited syntax for combining lexical items. Second, these stages are assumed to have adaptive value for cognition and communication. 


\subsection{Precursors?}

Did language emerge gradually from phylogenetic forerunners? To answer this question it is sometimes revealing to compare human languages with animal communication systems, including those - like birdsong - that are phylogenetically unrelated to human language. It is by now generally agreed that the language capacity is not a phylogenetic continuation of animal communication such as the gestural systems of nonhuman primates, though these are related to forms of nonlinguistic gestural and physiognomic communication. But these forms are all independent of the language capacity and the structure of UG. Moreover, no path leads from the ability to use a highly restricted repertoire of situationally dependent signals to the combinatorial capacity of human language. This assertion is unrelated to issues of modality, such as the question of whether, say, chimpanzees are better at visual or acoustic signals.

That said, the language capacity clearly has a prelinguistic basis. I already noted that the language capacity can build on the systems abbreviated as A-P and C-I (or on their predecessors, assuming that these systems have undergone change). But I want to emphasise that they are prerequisites rather than early instantiations of the language capacity, particularly with respect to selectional conditions.

\section{The origin of language}

From the Book of Genesis and Plato's speculations about whether the link between signifiers and signifieds is physei (natural) or thesei (conventional) to the speculations of Leibniz and Herder, the debate about language origin has focused on individual words. The origin of the language capacity is either taken for granted or confused with the origin of (the first) language. The combinatorial properties of human language have largely been ignored.

\subsection{Stages of language development}

The phylogenesis of UG and the origin of a language $\mathrm{L}$ are manifestly different issues. But neither can be understood in isolation. This is the upshot of section 3. In particular, the different versions of adaptation as well as the radical position of strict exaptation must all deal with the origin of a first 
language, which eventually activated the language capacity. We lack direct evidence about both language origin and the evolution of the language capacity. Nevertheless, the relevant facts regarding language origin fall within the range of linguistic theorising, whereas this is hardly the case with phylogenetic issues regarding the language capacity.

Following Bickerton (1995) and particularly Jackendoff (1999), in the remarks below I assume stages in the origin of language, stages that presumably interacted with the evolution of the underlying capacity. These stages do not correspond to modules of L - or of UG, as Pinker assumes -, but are closely related to the phenotype of language use. The scenario I describe below does not pretend to be a reconstruction of the actual developmental path, but represents a logical possibility.

The necessary principles of UG must be related to properties of linguistic expressions that could be (or in fact are) realised, given the constitutive condition of mapping Articulation to Conceptualisation. This yields a conceptually necessary set of properties that can be arranged in developmental stages. Two indispensable capacities are to be identified in this respect, whose characteristic prerequisites and consequences will be articulated in due course:

(18) Stimulus-free and situationally independent assignment of structured signals to conceptual representations (arbitrary sign formation).

(19) Systematic, recursive combination of signs into structures supporting compositional interpretation (compositionality).

Condition (18) roughly corresponds to Herder's notion of "reflection" as the source of language. It marks a decisive difference between human language and animal communication. In Bickerton's (1995) view, (18) and (19) represent two stages in the development of both the language capacity and of language. Condition (18) results in what Bickerton calls "protolanguage", which is transformed by the addition of (19) into modern human language. It might turn out, however, that arbitrary sign formation has the same condition of possibility as compositionality - in other words, that independence of stimulus control for basic expressions is tantamount to the capacity to combine them. I will leave the issue open, noting, though, that Jackendoff (1999) points out that modern languages exhibit fossilised cases of prelinguistic items like $s h$, hey, or ouch, which are dependent on specific situations and simultaneously devoid of syntactic status. 


\section{Manfred Bierwisch}

\subsection{Developing a lexical system}

Whereas exclamations like hey or wow are only appropriate in relevant situations, words like jump or dog do not depend on a dog or a jumping action being present. The crucial point is that a structure in C-I becomes accessible by a pattern in P-A. As I mentioned above, the relation on which this accessibility rests is the major topic in the history of attempts to account for the origin of language. There are three basic proposals.

First, Herder (1772) posits an act of naming caused by a salient feature of the thing to be identified. His example is a bleating sheep, which receives its name (internal bleating) by a sort of indexical relation. Second, Leibniz (1710) considers the act of naming to be based on the analogy between the shape of the name and the emotion induced by the perception of the object. His model postulates a synesthetic similarity between sound-pattern and object-sensation. The third proposal is that the links between words and meanings are conventional and arbitrary. All three semiotic types - indexical, iconic, and conventional signs - are involved in the mapping between A-P and C-I. Yet there can be little doubt that arbitrary signs comprise the overwhelming majority.

More important than the origin of individual words are the general conditions and consequences of the "protolexicon": the forerunner of proper lexical systems. I will address two of them.

First, the protolexicon does not have a strictly limited number of items. This distinguishes it from systems of primate calls and from all other prehuman sign systems (including the vocabulary of language-trained chimpanzees). This may or may not be a side effect of the capacity for stimulusfree sign use. The protolexicon's elasticity means that the system can expand incrementally. Its gradually increasing size and complexity may correspond to gradually increasing adaptive benefits. This avoids the theoretical pitfalls of adding up UG modules that lack independent useability. Moreover, once the protolexicon capacity is part of a population's genetic endowment, an expanding actual protolexicon can be transmitted as cultural rather than biological heritage.

Second, an increasingly large set of items cannot be accommodated as global, unstructured chunks of information, but only on the basis of systematic organisation that relies on structural dimensions or features of representation. This is true for aspects of both domains linked by the lexical system: conceptual relations in C-I and articulatory conditions in A-P. This raises the question of whether primitive elements of PF and SF are geneti- 
cally fixed prerequisites of the linguistic structure. In Bierwisch (2000), I have argued that UG need not provide fixed repertoires, but only general conditions, on which epigenetic development then constructs the actual primes via triggering experience. In any case, the protolexicon integrates two systems that become systematically structured in their own right. This is not a post-hoc effect, but an initial condition that first enables the formation of stimulus-free pairs of form and meaning.

The protolexicon's systematic nature seems to imply two other phenomena. The first is the initiation of Morphology. This involves formal features that are not (like phonetic features and semantic primes) directly based on A-P or C-I, but only have a status within the organisation of lexical items. Two stages can be identified. They build on each other logically, though not perforce developmentally. The examples below from English illustrate the two stages. Words like those in (20a) differ with respect to sex, but share the other conceptual conditions; no feature or segment in PF corresponds to the semantic distinction between male and female. By contrast, half the words in (20b) have the suffix -ess to mark the condition FEMALE; this might be said to reflect a distinction of SF in the makeup of PF.

(20) (a) son : daughter; boy : girl; uncle : aunt; king : queen; husband : wife

(b) actor : actress; prince : princess; duke : duchess; steward : stewardess

This type of partial systematisation also shows up in other human sign systems like colour-coding in traffic signs and the use of subscripts or superscripts in formal languages. For example, the terms of (21a) are replaced in (21b) and (21c) by more explicit notation:

$$
\text { (21) (a) } x, y, z, u, \ldots \text { (b) } x, x^{\prime}, ", x^{\prime \prime \prime}, \ldots \text { (c) } x_{1}, x_{2}, x_{3}, x_{4}, \ldots
$$

This means that $(20 \mathrm{~b})$ - like other arbitrary cases of derivation and inflection - relies on inherent properties of the capacity to organise nontrivial systems of pairs that relate form and meaning. The second stage emerges when in the correspondence /-es/ $\Leftrightarrow$ [FEMALE] the condition [FEMALE] (and the suffix related to it) acquire a formal, independent status. This status is directly accessible and supports the further systematisation of lexical elements. Once established, such a feature might be dissociated from the fixed interpretation in A-P and C-I. That is, [+ Feminine] can become a formal 


\section{Manfred Bierwisch}

feature of woman, girl, she, and so forth as well as of items (like ship names) where the conceptual condition related to FEMALE does not apply. My proposals do not ascribe a proper morphology to the protolexicon. They merely employ present-day phenomena to indicate that elements of the sort on which morphological systems rely are available as a side effect of the structural conditions necessary for burgeoning protolexical systems.

The second feature is presyntactic combination. The elements of the protolexicon allow this because they are stimulus-free and thus have an articulated structure. Due to PF's basic linearity, presyntactic combination can only amount to sequential juxtaposition. This might, however, lead to a structure in C-I that is more specific than that of the combined elements in isolation. The point is that we can imagine an elementary stage where elements of the protolexicon can be linked in the absence of specific combinatorial principles. Jackendoff notes fossilised residues of this in modern languages. For instance, compounds like steamboat, houseboat, and rowboat are formed by two elements linked conceptually according to our beliefs about the world, not according to syntactically based principles of compositionality.

One could speculate about whether recurrent juxtaposition, if it corresponds to conceptual conditions taken up in SF (like in find apple, eat apple, take apple, and have apple), provides the inherent foundation for nascent compositionality, a foundation that is comparable to the extraction of grammatical features described above. Without straining plausibility, I will merely note that there are surprising presuppositions and consequences connected to the capacity to create structured lexical items that are not controlled by situational dependence.

\subsection{Beyond protolanguage}

In a heavily debated report, Gopnik (1990) claims that certain grammatical deficits have genetic causes. Even if this is correct, we still don't know which aspects of the language capacity are genetically determined or how. The characteristics of Bickerton's "protolanguage" are thus speculative. This is of course equally true for the scenario I described above and for Jackendoff's (1999). But let's assume for a moment that the capacity to acquire and use protolexical knowledge is a precursor of the language capacity itself. The protocapacity can manifest itself in characteristic overt behaviour. Such behaviour consists of the use (and enables the accumulation) 
of shared knowledge in populations with a common heritage. But this is still different from the language capacity of recent homo sapiens. One could even argue that the big leap involves the change(s) that catapult the protolexicon capacity to UG. I will characterise three of its aspects. All are related to the central role of grammatical features, extending protolexical items $\langle\pi, \sigma\rangle$ into proper lexical items $\langle\pi, \gamma, \sigma\rangle$ discussed in section 2.1. One may, but need not, claim that this results from a single genetic condition emerging from a single phylogenetic change.

The first aspect is the formation of Phrase Structure described in (6) and (7). It involves two conditions. First, it provides the hierarchy or bracketing that goes beyond the mere juxtaposition of words and supports specific relations that can be drawn upon by compositional semantics as in example (9). In fact, it is needed to account for simple differences like those between (22)(a) and (b).

\section{(22) (a) [Mary [asked Bill] ] (b) [Bill [asked Mary] ]}

The second aspect is the categorisation of the constituents that accounts for the different properties of, say, his sleep (nominal head) and he sleeps (verbal head). In fact, only the integration of constituency and categorisation formulated in (7) creates UG's basic combinatorial aspect. Categorisation, however, is the core effect of formal features. This means that categories like Verb, Noun, and Determiner are just bundles of formal features. Their role is to organise the correspondence between PF and SF (see Wunderlich 1996 for an overview). It need not concern us here whether the relevant properties of features like [Referential] and [Functional] can be explained in a manner similar to those for [Feminine]. The point is that they determine a sort of second-order classification based on properties that do not derive directly from A-P or C-I, but from the correspondence to be established between these primary domains. The ability set up and use this second-order classification is a plausible candidate for the property that characterises the Big Leap.

The second aspect comprises morphological features like [Feminine]. This may well be related to the role of formal features more generally and may assign an independent, central status to second-order classification. Indeed, morphological categories like Gender, Number, Case, and Aspect should not be considered as supplements made in modern languages, supplements that could possibly be avoided for the sake of greater simplicity (Klein and Perdue 1997 strggest preeisely this on the basis of the "Basic 


\section{Manfred Bierwisch}

Variety" of second-language learners). Morphological categories play a central role in organising the PF-SF correspondence. Let me sketch three interdependent aspects of this central role.

First, it is well known that morphological features combine into rich inflectional and derivational systems. Even English, which has little inflectional morphology compared with, say, Hungarian or Georgian, displays feature combinations like (23). In these examples D stands for the category features of Determiners; Oblique and Plural stand for Case and Number, respectively:

(23) (a) /him/ [D; - Feminine, + Oblique, - Plural $]$

(b) $/$ she/ [D; + Feminine, - Oblique, - Plural $]$

(c) /them/ [D; + Oblique, + Plural $]$

Inflectional systems can display remarkable intricacy, a subject beyond the scope of this paper.

Second, the formal features are instrumental in establishing and regulating various types of grammatical relations imposed on the underlying Phrase Structure. One of them concerns the selectional constraints that relate lexical items to their complements, as illustrated in (24) and (25). The (semantically based) properties by which lexical items combine with complements are all specified by morphological features:

(24) (a) he dreams something

$\left(a^{\prime}\right)$ *he sleeps something

(b) he dreams of something

(b') *he sleeps of something

(25) (a) *his dream something

(b) his dream of something

As shown by (24a) and (24b), the Verb dream requires a Subject and alternatively accepts an Object or a Prepositional Phrase. By contrast, the Verb sleep is restricted to the Subject. As demonstrated by (25), the Noun dream accepts a Prepositional Phrase, but not an Object. These conditions are based on conceptual content: both dream and sleep require an Agent, but dream has a Theme, whereas sleep does not. Nevertheless, formal features mediate their realisation. Another relation marked by formal features is the concord that pronouns require for binding to their antecedent, as illustrated in (26), where her can refer to Mary in (26a), but not in (26b): 
(26) (a) Mary wants him to help her/*herself

(b) Mary wants to help her/herself

The third aspect depends on formal features in yet another respect. Chains of positions as in (27) (see section 2.3. above) are an extension of Phrase Structure that requires one constituent to be available in two structural positions:

(27) (a) I know, who he wants me to talk to.

(b) [C I know [C who [C he wants me [V to talk [P to ] ] ] ]

A chain's positions are bound together by formal features. Above, $[+\mathrm{Wh}]$ comes with the Interrogative who, which simultaneously characterises the embedded indirect question clause. Chomsky (1995) supposes this relation to be the result of copying (or attracting) a feature from a source to a target position (the whole constituent that contains the feature being carried along). In any case, the connection between chain positions crucially depends on the availability of formal features. In addition, chain formation is ubiquitous in natural language and reconciles multiple requirements that cannot be met at the same structural position. Though it complicates the (apparently) simplest possible correspondence between PF and SF, it enables the discrepancies between conflicting conditions in Phrase Structure to be overcome. Finally, chain formation is a proper amendment to bare Phrase Structure. Like Phrase Structure and Morphology, it draws on formal features, but might result from a separate step in the evolution of UG.

In this paper I have commented on the following aspects of UG via which the language capacity goes beyond the protolexical capacity and underlies stages in language origin:

(28) (a) Free disposal of formal features, defining syntactic and morphological categories;

(b) Phrase Structure, integrating general hierarchy formation with category assignment;

(c) Morphological systems that make grammatical features accessible for selectional restrictions, agreement, concord (that is, relations that enrich Phrase Structure);

(d) Formation of Chains of structural positions connected by formal features. 


\section{Manfred Bierwisch}

One can emphasise aspects or components of the language capacity in slightly different ways. For instance, the important role played by Argument Structure and the linking of lexical heads to appropriate Complements are subsumed here under the selectional restrictions in (28c). One could easily claim a central position for this momentum. Delimiting components and subcomponents is thus a matter of argument. My remarks have of course been incomplete. I did not, for instance, say anything about the conditions that support the abstraction of elements and relations of SF from C-I. Without these, neither contrasts like behind the wall (location) and after the wall (time) nor the vast area of metaphors can be accounted for. Nevertheless, it is obvious that (28) (b) (c), and (d) are logically dependent on (28a). It addresses the centrality of formal features, which are - differing from phonetic and semantic primes - a kind of currency whose value is only fixed internally and due to the computational system that determines the correspondence between PF and SF. It is not obvious whether Categorisation in (28b) and Morphology (28c) are independent of each other. But it is clear that the complexity of Chain formation (28d) presupposes (28) (a) to (c). According to Borer and Wexler (1987), it is a prime candidate for later maturation into ontogenetic development. Perhaps it is also a relatively late addition phylogenetically.

\section{Three concluding remarks}

First, attempting to sort out structural conditions like in (28) above by no means implies that components of G (or UG) correspond to separate neurophysiological counterparts. Kean (1992) points out that there is no direct and discrete representation of grammatical components in the brain. Moreover, this undermines speculations about simple relations between UG's properties and their genetic foundation. Yet if, as Kean suggests, a distinction can be made between brain systems for representation and those for re-representation of knowledge, then linguistic knowledge crucially depends on conditions of re-representation that mediate the primary representations of A-P and C-I. In this view, the availability of formal features and the options based on them would be among the language capacity's conditiones sine qua non because re-representation seems to be the most appropriate way of characterising the essence of formal features. 
Second, I have proposed how to avoid the paradox of language evolution by combining two distinct but interrelated problems, both of which have to be solved anyway: the evolution of the language capacity and the origin of linguistic knowledge. These frequently confounded issues must be clearly distinguished because they depend on fundamentally different conditions affecting the genetic heritage as well as possible knowledge based on it. But it seems that a plausible scenario emerges if they are construed to depend on each other in a non-vicious circle. The capacity to accumulate lexical items could gradually lead to a developmental stage where a random variation indeed leads to an improvement of the linguistic capacity, justifying the urgently desired selectional benefit. The stages in this type of scenario of course leave us with various unanswered questions. Suppose for a moment that Herder was basically right to suggest that "reflection" is the condition of possibility for the initial stage's stimulus-free naming. Is the combinatorial potential thus an implicit condition whose importance Herder failed to recognise? Or must we posit separate evolutionary steps that are not involved in building up comparatively complex protolexical items? In any event, it is clear that the accumulation and transmission of (proto) lexical knowledge relies on the social (communicative) dimension rather than on the strictly cognitive dimension in the development of the language capacity.

Finally, the scenario's plausibility presupposes that the language capacity's evolution was gradual and that it relied on adaptive selection. This is by no means certain. In the end, perhaps Chomsky is right to have doubts about the adaptive explanation. In the terms of one of Murphy's Laws: for any complex problem there exists a solution that is simple, plausible, and wrong. 